ISSN 0373-580 X

Bol. Soc. Argent. Bot. 49 (3): 405-415. 2014

\title{
NOVEDADES Y NOTAS TAXONÓMICAS EN ESPECIES DE SENECIO de la PATAgonia, ARgentina
}

\author{
ROBERTO D. TORTOSA ${ }^{1,2}$ y ADRIANA BARTOLI
}

\begin{abstract}
Summary: Novelties and taxonomical notes in Senecio from Patagonia, Argentina. A new species and three new varieties of the genus Senecio L. sect. Senecio, are described. The new species, S. pichineuquensis Tortosa \& Adr. Bartoli, included in the series Xerosenecio (Cabrera) Cabrera \& S. E. Freire, has a geographic distribution in the south of Mendoza and north of Neuquén Provinces. It is characterized by ligneous rigid stems with long internodes, linear, rigid leaves, few-flowered discoid yellow heads grouped in dense corymbs, and narrowly cylindrical involucre. A key is provided for its differentiation from the allied species. A variety of this species with cream-color heads, S. pichineuquensis var. albus Tortosa \& Adr. Bartoli, is described. The other two new varieties, S. bracteolatus Hook. et Arn. var. luteous Tortosa \& Adr. Bartoli and S. varvarcensis Cabrera var. pinnatus Tortosa \& Adr. Bartoli, belong also to the series Xerosenecio, and are apparently endemic to the north of Neuquén Province. The variety luteous of $S$. bracteolatus differs from the typical variety by its gold yellow florets, higher narrowly cylindrical involucre, and lower number of phyllaries and flowers, whereas the variety pinnatus of $S$. varvarcensis may be distinguished from the typical variety by its pinnate leaves (vs. entire leaves in var. varvarcensis). Two other species of Senecio that inhabit in Patagonia are included in the synonymy of different species: S. subdiscoideus Sch. Bip. ex Wedd. of the series Metazanthi (Meyen) Cabrera et S. E. Freire, as synonym of S. kingii Hook. f., and S. bipontinii Wedd. of the series Chilenses DC. ex M. G. López, A. F. Wulff \& Xifreda subseries Caespitosi (O. Hoffm.) Cabrera et S. E. Freire as synonym of S. crithmoides Hook. et Arn. S. crithmoides, S. iberidifolius Phil., and S. purpuratus Phil. are lectotipified.
\end{abstract}

Key words: Argentina, Asteraceae, Senecio.

Resumen: Se describen una nueva especie y tres nuevas variedades en el género Senecio L. sect. Senecio. La nueva especie, S. pichineuquensis Tortosa \& Adr. Bartoli, que se incluye en la serie Xerosenecio (Cabrera) Cabrera \& S. E. Freire, se distribuye en el sur de la provincia de Mendoza y en el norte de la provincia de Neuquén. Se caracteriza por sus tallos leñosos rígidos con largos entrenudos, hojas lineares también rígidas, capítulos amarillos con pocas flores, agrupados en densos corimbos, e involucro angostamente cilíndrico. Se incluye una clave para diferenciarla de las especies afines. Se describe además, para esta especie, una variedad de capítulos blanco-cremosos, S. pichineuquensis var. albus Tortosa \& Adr. Bartoli. Las otras dos nuevas variedades, S. bracteolatus Hook. et Arn. var. luteous Tortosa \& Adr. Bartoli y $S$. varvarcensis Cabrera var. pinnatus Tortosa \& Adr. Bartoli, pertenecen también a la serie Xerosenecio, y son aparentemente endémicas del norte de la provincia de Neuquén. La variedad luteous de $S$. bracteolatus difiere de la variedad típica por el color amarillo oro de sus flores, el involucro angostamente cilíndrico y de mayor altura, y por el menor número de filarios y flores, mientras que la variedad pinnatus de $S$. varvarcensis se distingue por sus hojas pinnadas (vs. hojas enteras en la var. varvarcensis). Otras dos especies de Senecio que viven en Patagonia, se incluyen como sinónimo de diferentes especies: S. subdiscoideus Sch. Bip. ex Wedd. de la serie Metazanthi (Meyen) Cabrera et S. E. Freire, en la sinonimia de S. kingii Hook. f. y S. bipontinii Wedd. de la serie Chilenses DC. ex M. G. López, A. F. Wulff \& Xifreda subserie Caespitosi (O. Hoffm.) Cabrera et S. E. Freire, en la de S. crithmoides Hook. et Arn. Se designan lectotipos para $S$. crithmoides, S. iberidifolius Phil. y S. purpuratus Phil.

Palabras clave: Argentina, Asteraceae, Senecio.

Facultad de Agronomía, Universidad de Buenos Aires, Av. San Martín 4453, 1417 Buenos Aires, Argentina. tortosa@agro.uba.ar; cbartoli@agro.uba.ar

2 Consejo Nacional de Investigaciones Científicas y Técnicas. 


\section{INTRODUCCIÓN}

Durante el e studio de la s especies de Senecio sección Senecio de Patagonia, hallamos algunos ejemplares del sur de la Provincia de Mendoza y del norte de la provincia de $\mathrm{N}$ euquén, con capítulos discoides amarillos, que parecían constituir una nueva especie para la ciencia.

Con el propósito de coleccion ar esos materiales, realizamos un viaje a 1 os departamentos de Ch os Malal y Minas, del norte de la Provincia de Neuquén ( 1). En esa pequeña área crecen 32 es pecies de Senecio, de un total de 122 especies que habitan la Patagonia argentina. Cinco de ellas co nstituyen endemismos de di cha región: $S$. breviramulus Tortosa et Adr. Bartoli, S. covuncensis Cabrera, $S$. divaricoides Cabrera, S. sandwithii Cabrera y $S$. varvarcensis Cabrera, y otros $7 \mathrm{t}$ axones son endémicos de es ta región y zonas vecinas de la provincia de Mendoza y de Chile: S. aspericaulis J. Rémy, S. chilensis Less. fo. discoideus Cabrera, $S$. covasii Cabrera, S. depressus Hook. et Arn., S. leucophyton Phil., S. pumilus Tortosa et Adr. Bartoli y $S$. tricephalus Kuntze.

Durante el viaje mencionado hallamos varias poblaciones de 1 a presunta nueva especie, una variedad para la $\mathrm{m}$ isma con capítulos blancocremosos, ejemplares de $S$. varvarcensis con hojas pinnadas y algunas poblaciones de $S$. bracteolatus con capítulos de mayor tamaño que el conocido para la especie y con flores de color amarillo oro.

En el presente trabajo describimos las novedades, e incluimos, como Notas Taxonómicas, el pasaje de dos especies patagónicas, Senecio subsidcoideus Sch. Bip. ex Wedd. y S. bipontinii Wedd., a la sinonimia de $S$. kingii Hook. f. y $S$. crithmoides Hook. et Arn. respectivamente.

\section{MATERIALES Y MÉTOdOS}

Según los casos se estudiaron los materiales a campo, se consultaron ejemplares de herbario y en la medida de 1 o posible se examinaron los tipos nomenclaturales.

\section{REsultados}

1. UNA NUEVA ESPECIE DE SENECIO SER. XEROSENECIO CON UNA VARIEDAD
Senecio pichineuquensis Tortosa. \& Adr. Bartoli, sp. nov. TIPO: Argentina. Neuquén: Dpto. Minas, entre Manzano Amargo y Pichi Neuquén, a la salida de Manzano Amargo, 36 $42^{\prime} \mathrm{S}, 70^{\circ} 46^{\prime}$, 17-II- 2010, R. D. Tortosa 7/10 (holotipo, BAA; isotipos, CTES, K, LP, MO, SI). Fig. 2.

Inter species Senecionis seriei Xerosenecionis (Cabrera) Cabrera et S. E. Freire caulibus rigidis intenodiis longis, foliis linearibus rigidis sublanuginosis, capituliis numerosis, paucifloris, et involucro anguste cylindrico 7.5--8 mm alto differt.

Arbustos de 0,6-1,4 m alt., muy ramificados en la base; tallos leñosos, erectos, rígidos, con internodios largos, estriados, con la porción basal albolanuginosa y la p orción distal verde-amarillenta, glabra o subglabra. Hojas alternas, sésiles, linear o linear-elípticas (raro las hojas basales elípticas), con ápice agudo, de $2-4$ × $2 \mathrm{~cm}$, margen involuto, enteras o con algunos dientes, albo-lanuginosas cuando jóvenes, subglabras o gl abras cuando adultas. Capítulos discoides, agrupados de 10 a 20 en el extremo de 1 as ramas; pedúnculos de $0,7 \mathrm{~cm}$; involucro angostamente cilíndrico, de 6-8 X 3-3,5 $\mathrm{mm}$, caliculado. Brácteas del calículo 6-7, elípticas, de ca. $2 \mathrm{~m} \mathrm{~m}$ long., densamente albo-lanuginosas. Filarios 8-9, subglabros a lanuginosos en el dorso y densamente albo-lanuginosos en la base, con el ápice acuminado, oscuro. Flores 11 a 25 por capítulo, hermafroditas, con corola tubulosa, glabra, de 6-7 mm long., con lóbulos triangulares de 0,7 mm long.; anteras 1,5-2 mm; ramas del estilo de ca. 0,6 $\mathrm{mm}$. Aquenios cilíndricos, glabros, de 4-5 mm; papus blanco de 6-6,5 $\mathrm{mm}$.

Etimología. El epíteto específico pichineuquensis hace referencia a la localidad $\mathrm{P}$ ichi Neuquén, que toma su nombre del pequeño río homónimo, tributario del río Neuquén. En idioma mapuche pichi significa pequeño (Perón, 2000), y pichi-Neuquén, pequeño Neuquén.

Categoría en la Lista Roja de IUCN. Debido al área de distribución y abundancia esta especie debe ser considerada en la ca tegoría de $\mathrm{P}$ reocupación Menor (LC) de acu erdo con los criterios de IUCN (IUCN, 2001).

Obs. Senecio pichineuquensis se incluye en la serie Xerosenecio (Cabrera) Cabrera \& S. E. Freire subseries Filaginoidei (C abrera) Cabrera et S. E. Freire, y es afín a $S$. caricifolius Hook. et Arn., $S$. 


\section{R. D. Tortosa y A. Bartoli. - Novedades en el género Senecio}

filaginoides DC., S. neaei DC. y $S$. patagonicus Hook. et Arn., por s u hábito subarbustivo, y sus tallos erectos, hojas lineares a an gostamente elípticas, capítulos discoides agrupados en el extremo de tallos hojosos e involucro igual o menor a $8 \mathrm{~mm}$ de longitud. La nueva especie se distingue de ellas p or su involucro angostamente cilíndrico, los tallos con la p orción basal albo-lanuginosa y la parte distal amarillenta, glabra o subglabra, con entrenudos largos y las hojas lanuginosas cuando jóvenes.

\section{CLAVE PARA DIFERENCIAR SENECIO PICHINEUQUENSIS Y SUS ESPECIES AFINES DE LA PATAGONIA}

1a. Hojas angostamente lineares, menores de $1 \mathrm{~mm}$ lat. Involucro de 4.5--6 mm alt.

2a. Involucro obcónico. Filarias persistentes después de la madurez de los aquenios. Capítulos con 16 a 20 flores.

Senecio neaei

2b. Involucro cilíndrico. Filarias deciduas después de la madurez de los aquenios. Capítulos con 22 a 26 flores.

Senecio caricifolius

1b. Hojas lineares o linear-elípticas, mayores de $1 \mathrm{~mm}$ lat. Involucro de 6--8 $\mathrm{mm}$ alt.

3a. Involucro acampanado. Capítulos con 25 a $50 \mathrm{f}$ lores. Tallos y hojas densamente lanuginosas con porciones subglabras.

Senecio patagonicus

3b. Involucro cilíndrico o angostamente cilíndrico. Capítulos con 11 a 25 flores

4a. Tallos y hojas densamente albo-lanuginosos. Filarios 8-10(-13)

Senecio filaginoides

4b. Tallos albo-lanuginosos solo en la porción basal, la porción distal subglabra o g labra y verde amarillenta. Hojas adultas glabras o subglabras. Filarios 6-7.

Senecio pichineuquensis

\footnotetext{
1.1. Senecio pichineuquensis var. del sur de la provincia de Mendoza y norte de la pichineuquenisis provincia de Neuquén (Argentina), a elevaciones de 1300-1550 m. Crece en suelos rocosos asociada con

Capítulos con flores amarillas, involucro de 6-8 Senecio breviramulus Tortosa \& Adr. Bartoli, S. mm alt. bracteolatus var. luteus, $S$. varvarcensis var. pinnatus y con especies de Adesmia DC., Mulinum

Distribución y há bitat. La variedad típica de
} Senecio pichineuquensis fue hallada en las montañas 
Material adicional examinado: ARGENTINA. Mendoza: Dpto. Malargüe, pozo de las Ánimas, 12I-1971, s. col., ex Instituto de Geografía, U. N. C. $n^{\circ}$ 3 (SI); Río Grande, $45 \mathrm{~km}$ al N de Las Choicas, 22I-1982, A. L. Cabrera et al. 33486 y 33488 (SI). Neuquén: Dpto. Minas, Pichi-Neuquén, valle del río Pichi-Neuquén, 22-I-1970, O. Boelcke 13640 (BAA); el llano de L a Primavera, 12-I-1993, González 985 (LP); al n orte de Varv arco, 28-III2009, A. Moore et al. 50 (BAA, SI) y 52 (BAA, LP); de La Primavera hacia Andacollo, 17-II-2010, R. D. Tortosa 2/10 (BAA, SI); de Andacollo a Las Ovejas, 17-II- 2010, R. D. Tortosa 3/10 (BAA, MO); de Andacollo a Las Ovejas, a $4 \mathrm{~km}$ de Las Ovejas, 17II- 2010, R. D. Tortosa 4/10 (BAA) y 6/10 (BAA, CTES, LP, MO); en tre Manzano Amargo y Pichi Neuquén, 17-II- 2010, R. D. Tortosa 11/10 (BAA, $\mathrm{K}, \mathrm{MO}$ ) y $12 / 10$ (BAA, CTES, MO); a $4 \mathrm{~km}$ de Varvarco hacia Manzano Amargo, 18-II-2010, R. D. Tortosa 14/10 (BAA, SI) y 15/10 (BAA); llegando a Las Ovejas desde Varvarco, 18-II-2010, R. D. Tortosa 18/10 (BAA) y 19/10 (BAA); de Las Ovejas hacia Andacollo, 18-II-2010, R. D. Tortosa 21/10 (BAA, SI); ca mino de L as Ovejas a lag una Epulafquen, a $1 \mathrm{~km}$ del desvío a Las Lagunas, 19-II2010, R. D. Tortosa 23/10 (BAA, BAB), 24/10 (BAA), 26/10 y 27/10 (BAA); camino de Las Ovejas a laguna Epulafquen, a 2,5 $\mathrm{km}$ del desvío a L as Lagunas, 19-II-2010, R. D. Tortosa 30/10 (BAA, $\mathrm{MO})$; camino de Las Ovejas a laguna Epulafquen, a $5 \mathrm{~km}$ del desvío a Las Lagunas, 19-II-2010, R. D. Tortosa 33/10 (BAA); Laguna Epulafquen, 19-II2010, R. D. Tortosa 38/10 (BAA, LP). Dpto. Chos Malal, estancia Chacaico, 20-XII-1992, González Arzac 954 (LP).

1.2. Senecio pichineuquensis var. albus Tortosa \& Adr. Bartoli, var. nov. TIPO. Argentina. Prov. Neuquén: Dpto. Minas, Andacollo, a los costados del puente próximo a la Escuela Huarco, 10-XII2010, A. Bartoli y R. D.Tortosa 27/10 (holotipo, BAA).

A varietate typica Senecionis pichineuquensis haec varietas differt capitulis majoribus et flo ribus albo-cremeis.

Capítulos con flores blanco-cremosas, involucro de ca. $9 \mathrm{~mm}$ alt.

Distribución y hábitat: Esta variedad fue hallada en el norte de la provincia de Neuquén, conviviendo

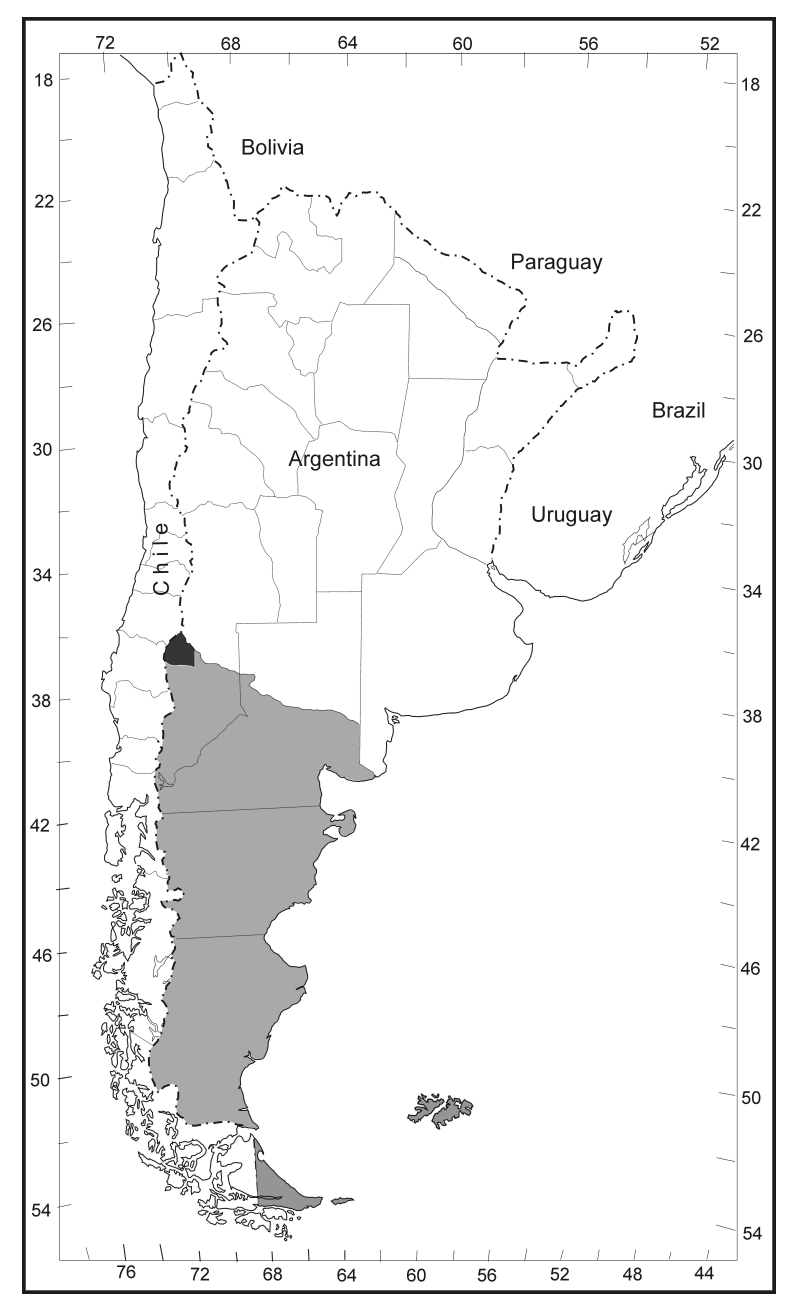

Fig. 1. Ubicación de los departamentos Chos Malal y Minas de la provincia de Neuquén (en negro), en el norte de la Patagonia (grisado).

a veces con la $\mathrm{v}$ ariedad típica $\mathrm{y}$ otras veces formando poblaciones puras.

Material adicional examinado: ARGENTINA: Neuquén: Dpto. Chos Malal, entre La Salada y Chapua, 13-XII-2010, A. Bartoli y R. D . Tortosa 43/10 (BAA).

\section{UNA NUEVA VARIEDAD DE SENECIO BRACTEOLATUS}

Senecio bracteolatus Hook. et Arn. J. Bot. (Hooker) 3: 347. 1841. TIPO: Argentina. Buenos Aires (como Buenos Ayres, procedencia sin duda errónea), J. Gillies s. n. (holotipo, K [K00032051], imagen digital!; isotipo, $\mathrm{K}$ [K000497950], imagen digital!). 
R. D. Tortosa y A. Bartoli. - Novedades en el género Senecio

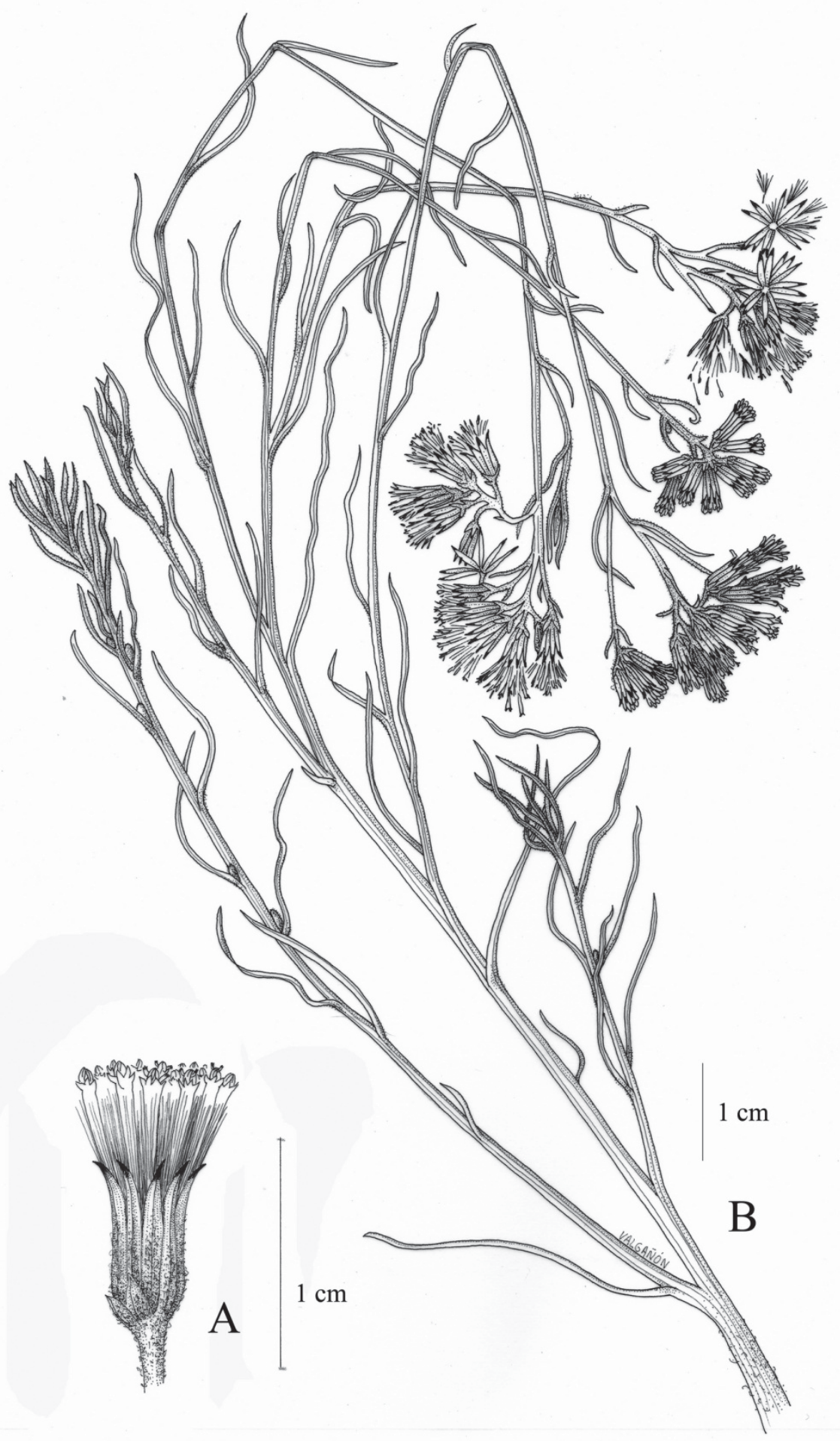

Fig. 2. Senecio pichineuquensis. A. Capítulo. B. Hábito (holotipo R. D. Tortosa 7/10). 
=Senecio coxi Phil., Anales Univ. Chile 23: 468. 1863. TIPO: Argentina. Pampa de Patagonia 186263, Cox s. n. (holotipo, SGO 60661, imagen digital!; isotipo SI [SI001039], imagen digital!).

Arbusto de 0,3-1 m alt., glabro. Tallos erectos, ramificados en la base, hojosos hasta el ápice. Hojas sésiles, algo crasas, lámina angostamente linear, de 1,5-4,5 cm x 0,5-1 mm, margen entero. Capítulos discoides, numerosos en el extremos de las ramas. Involucro caliculado; bracteolas del calículo elípticas, de 2-2,5 mm. long. Filarios elípticos, glabros, más o menos glandulosos. Flores tubulosas, amarillas. Aquenios cilíndricos, glabros o pubescentes. Papus blanco.

\subsection{Senecio bracteolatus var. bracteolatus}

Nombre vulgar: "charcao verde"

Flores amarillo-pálido. Involucro cilíndricoacampanado, de 5-6,5 X 3,5-4 mm. Filarios 9-13. Flores 16-24(30).

Iconografia: Cabrera, 1971: 248, fig. 251.

Distribución y hábitat: Vive en Argentina, desde la el norte de la provincia de Neuquén hasta el noreste de la provincia de Chubut.

2.2. Senecio bracteolatus var. luteus Tortosa \& Adr. Bartoli, var. nov. TIPO: Argentina. Neuquén: Dpto. Minas, entre Manzano Amargo y Pichi Neuquén, $36^{\circ} 42^{\prime} \mathrm{S}, 70^{\circ} 46^{\prime} \mathrm{W}$, 17-II- 2010, R. D. Tortosa 10/10 (holotipo, BAA; isotipos, LP, MO). Fig. 3.

A varietate typica Senecionis brateolatus Hook. et Arn. haec varietas capitulo aureo involucro anguste cylindrico 7-9,5 mm alt., minus phyllariis et floribus differt.

[1]Flores amarillo-doradas. Involucro angostamente cilíndrico, de 7-9,5 X 2,5-3 mm. Filarios 7-10. Flores 14-16.

Distribución y hábitat. Fue hallada en el norte de la provincia de $\mathrm{N}$ euquén, a $2000 \mathrm{~m}$ s. m. Habita suelos arenosos y rocosos, formando pequeñas poblaciones, junto con Senecio pichineuquensis, Haplopappus grindelioides DC., Quinchamalium chilense Molina y Chuquiraga sp.
Categoría en la Lista Roja de IUCN. Debido a su área de distribución reducida, y al tamaño pequeño de sus poblaciones, esta variedad debe s er considerada en Peligro Crítico (CR) de acuerdo con los criterios de IUCN (IUCN, 2001).

Etimología. El nombre luteous hace referencia al color amarillo brillante de sus flores.

Material adicional examinado: ARGENTINA. Neuquén: Dpto. Chos Malal, entre Los Menucos y arroyo Aquihueco, 29-III-2009, A. Moore et al. 65 (BAA); arroyo Aquihueco, 29-III-2009, A. Moore et al. 66 (BAA); entre Chos Malal y Andacollo, 20-II2010, R. D. Tortosa 42/10 (BAA, K). Dpto. Minas, entre Manzano Amargo y Pichi Neuquén, 17-II2010, R. D. Tortosa 9/10 (BAA, LP) y 13/10 (BAA, $\mathrm{SI})$; camino de Las Ovejas a laguna Epulafquen, a 5 $\mathrm{km}$ del desvío a L as Lagunas, 19-II-2010, $R$. D. Tortosa 32/10 (BAA, CTES).

\section{UNA NUEVA VARIEDAD DE SENECIO VARVARCENSIS}

Senecio varvarcensis Cabrera, Fl. Patagónica 7: 249. 1971. TIPO: Argentina, Neuquén, Dpto. Minas, Varvarco Campos, 28-I-1970, O. Boelcke 13999 (holotipo, BAA [BAA00001223]!, isotipos BAB[BAB00000124]!, LP [LPLP000701]!, SI!).

Sufrútice 0,3-0,4 m alt., glabro, áspero glanduloso. Tallos erectos, poco ramificados en la base, hojosos hasta el ápice. Hojas sésiles. Capítulos discoides, dispuestos 2 a 6 e $n$ cimas corimbiformes en el ex tremos de las ra mas. Involucro cilíndricoacampanado, caliculado, de 8-9 X $5 \mathrm{~mm}$; bracteolas del calículo pocas, lineares, cortas. Filarios 13-15, lineares, glabros, áspero-glandulosos. Flores 18-24, amarillas. Aquenios glabros. Papus blanco.

\subsection{Senecio varvarcensis var. varvarcensis}

Hojas simples angostamente lineares, de 2,5-3,5 cm x 0,5, margen entero, involuto.

Iconografía: Cabrera, 1971: 248, fig. 252.

Distribución y hábitat. Sólo hallada en el norte de Neuquén, departamento Minas.

3.2. Senecio varvarcensis var. pinnatus Tortosa \& Adr. Bartoli, var. nov. TIPO: Argentina. Neuquén: Dpto. Minas, camino de Las Ovejas a laguna Epulafquen, a $2,5 \mathrm{~km}$ del desvío a $\mathrm{L}$ as 
R. D. Tortosa y A. Bartoli. - Novedades en el género Senecio

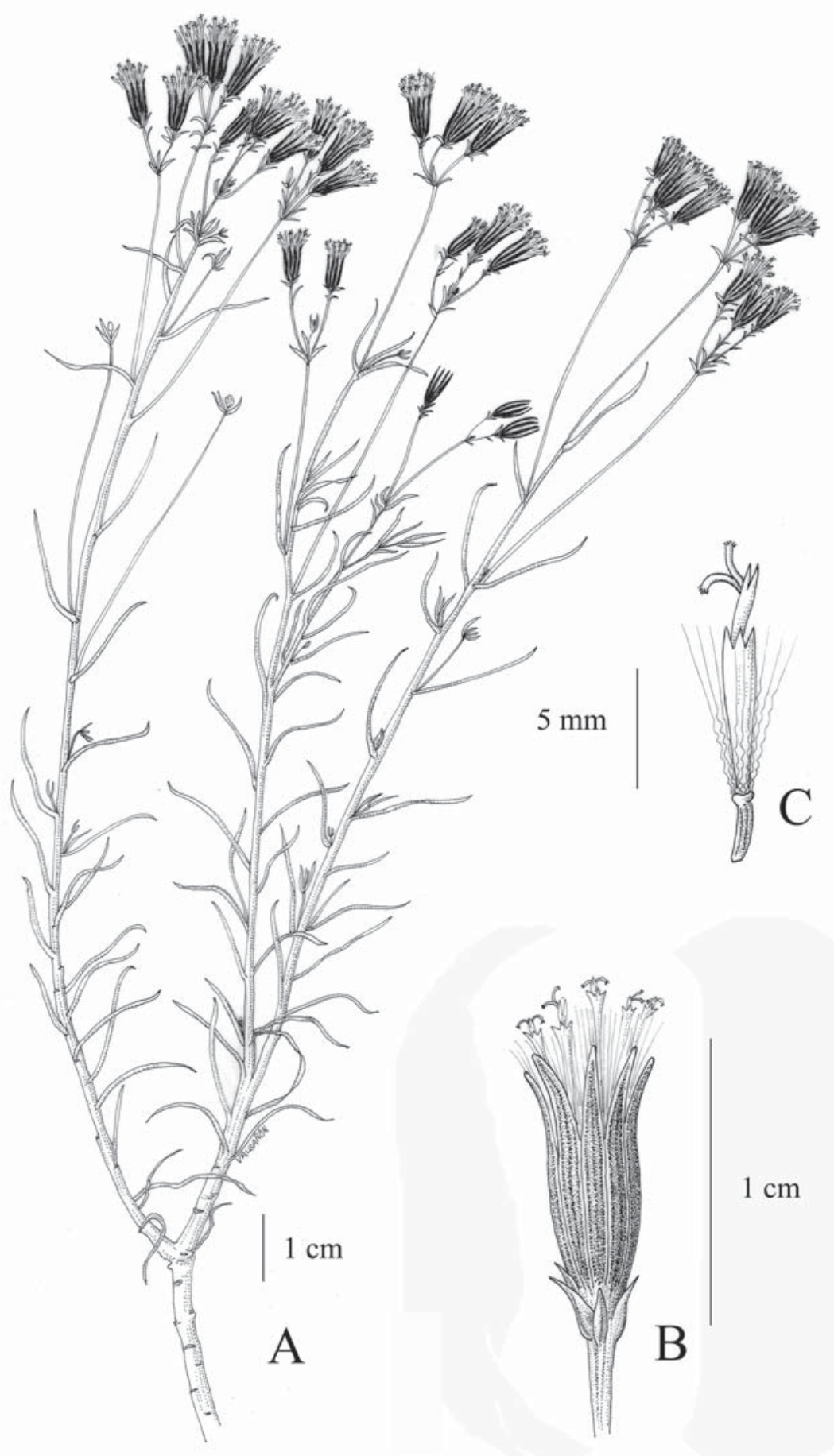

Fig. 3. Senecio bracteolatus var. luteus. A. Hábito. B. Capítulo. C. Flor (holotipo R. D. Tortosa 10/10). 
Lagunas, $37^{\circ} 01^{\prime} \mathrm{S}, 70^{\circ} 50^{\prime} \mathrm{W}, 19-\mathrm{II}-2010, R$. D. Tortosa 31/10 (holotipo, BAA; isotipos K, LP, MO, SI). Fig. 4.

A varietate typica Se necionis varvarcensis Cabrera haec varietas foliis pinnatis differt.

Hojas pinnatisectas con 1 o 2 pares de segmentos lineares, de hasta $2,5 \mathrm{~mm}$ long., algunas hojas distales simples.

Distribución y hábitat. Hallada en el norte de la provincia de Neuquén, a 1300-1550 m s. m. Habita en suelos pedregosos y fue siempre encontrada asomando sus capítulos entre matas de Mulinum Pers., que se comportan como plantas nodrizas. Convive con Chuquiraga Juss. y Senecio pichineuquensis.

Categoría en la Lista Roja de IUCN. Debido a su área de distribución reducida, y al tamaño pequeño de sus poblaciones, esta variedad debes er considerada en Peligro Crítico (CR) de acuerdo con los criterios de IUCN (IUCN, 2001).

Etimología. El nombre pinnatus hace referencia a las forma de las hojas.

Material adicional examinado: ARGENTINA. Neuquén: Dpto. Minas, de L as Ovejas a $\mathrm{C}$ hos Malal, 28-III-2009, A. Moore et al. 59 (BAA, CTES, K, MO); ca mino de Las Ovejas a la guna Epulafquen, a $2.5 \mathrm{~km}$ del desvío a Las Lagunas, 19II-2010, R. D. Tortosa 29/10 (BAA, CTES, K, MO, SI).

\section{NOTAS TAXONÓMICAS}

\subsection{SENECIO SUBDISCOIDEUS SINÓNIMO DE $S$. KINGII}

Cabrera (1971) consideró especies válidas a Senecio kingii Hook. f. y S. subsidcoideus Sch. Bip. ex Wedd., a mbas de 1 a serie Metazanthi (Meyen) Cabrera et S. E. Freire, dif erenciándolas por s us hojas (dentadas o l obadas en la primera y profundamente bipinatisectas en la última) y por el calículo (formado por pocas brácteolas, cortas, en la primera y con muchas, alcanzando en longitud a la mitad del involucro, en la segunda).

Ya en 1915, H osseus notó la correspondencia entre ambas species y consideró a $S$. subdiscoideus una forma de S. kingii.
La forma de las hojas en S. kingii presenta gran variación -ya señalada por Tortosa \& Bartoli (2010) al incluir en su sinonimia a S. gymnocaulus Phil.-, la que se manifiesta no sólo en la forma de la lá mina sino también en la profundidad de las incisiones; lo mismo ocurre con el número y tamaño de 1 as bracteolas. Por lo antedicho entendemos que $S$. subdiscoideus debe considerarse sinónimo de $S$. kingii.

Senecio kingii Hook. f., Fl. Antarct. 2: 314. 1846. TIPO: Chile. Port Famine (Puerto Hambre), Capt. King (holotipo, K [K000497964], imagen digital!).

= Metazanthus grandiflorus Meyen, Reise 1: 356. 1834. TIPO : Chile. Santiago : Río Maipo, III-1831, Meyen s. $n$. (B, destruído en la 2a. g uerra, nom. illeg., non Berg.; foto (F), F0BN015601).

= Senecio subdiscoideus Sch. Bip. ex Weddell, Chlor. Andina 1: 121. 1856. Senecio kingii fo. subdiscoideus (Sch. Bip ex Weddell) Hosseus, Trab. Inst. Bot. Farmacol. 33: 87. 1915.. Senecio purpuratus var. subdiscoideus (Sch. Bip ex Weddell) Reiche, Anales Univ. Chile 114: 150. 1904. TIPO: Chile. In cucumine altissimo Cordillera de Ranco ad nives aeternas, X II-1854, W. Lechler 2904 (holotipo, P [P01816482], imagen digital!; isotipos, GH, [GH00012208] imagen digital!; K [K000497960], imagen digital!; NY [NY00259420], imagen digital!, P [P01816483] imagen digital! y [P01816484] imagen digital!). Syn. nov.

= Senecio purpuratus Phil., Linnaea 28: 741. 1858. LECTOTIPO, aquí designado: Chile. Cordillera de Linares, I-1856, Germain s. n. (SGO; isolectotipos: HAL [HAL0112662] imágen digital!, LP [LP002489]!). Syn. nov.

= Senecio caespitosus Phil., 1.c. TIPO: Chile. Aysén : Cerro Doce de F ebrero, F. Fonk s. n. (no visto). Syn. nov.

= Senecio breviculus Phil., Anales Univ. Chile 21: 398. 1862. TIPO : Argentina. Portillo, lado de Mendoza, 1861/62, W. Diaz s. n. (isotipo, LP [LP002350]!). Syn. nov.

= Senecio gymnocaulos Phil., Anales Univ. Chile: 88: 23. 1894. TIPO: Argentina. Neuquén: Lago Lácar, II-1887, O. Philippi s. n. (holotipo, SGO; isotipo, LP [LP002388]!). 
R. D. Tortosa y A. Bartoli. - Novedades en el género Senecio

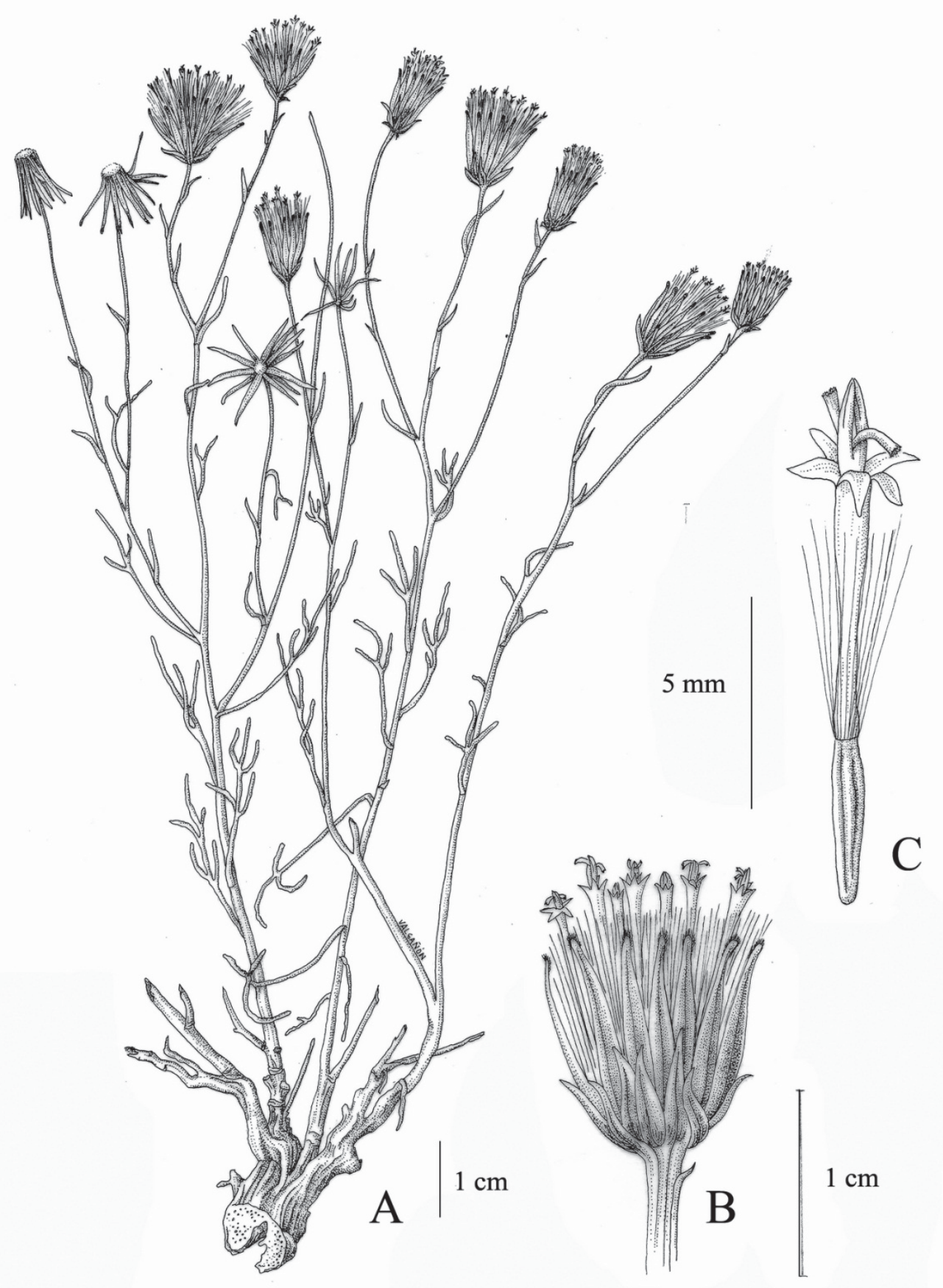

Fig. 4. Senecio varvarcensis var. pinnatus. A. Hábito. B. Capítulo. C. Flor (holotipo R. D. Tortosa 31/10). 
= Senecio kurtzii Albov, Revista Mus. La Plata, Secc. Bot. 7: 739. 1896. Nom. illeg. (non S. kurtzii Phil.1894). Senecio paradoxus Albov ex Kurtz, Bol. Acad. Nc. Ci. 16: 235. 1900 (n uevo nombre para $S$. kurtzii Albov). Senecio kingii var. paradoxus (Albov ex Kurtz) Cabrera, Lilloa 15: 83. 1949. TIPO: Argentina. Tierra del Fuego: Ushuaia, $N$. Alboff 551 (holotipo, LP [LP002408]!).

= Senecio poculiferus S. Moore ex Rendle, J. Bot. 42: 375. 1904. TIPO: Argentina. Santa Cruz: Lago Argentino, Península Burmeister, 1900-1901, H. H. Prichard (holotipo, BM, no visto).

Iconografia: Cabrera, 1949: 85, fig. 16 (s ub. Senecio gymnocaulos); Cabrera, 1971: 230, fig. 230 (sub. S. subdiscoideus), fig. 231 (sub. S. kingii).

Distribución y hábitat. Vive en Argentina desde la provincia de $\mathrm{S}$ an Juan hasta Tierra del Fuego, desde los 130 a los $3800 \mathrm{~m} \mathrm{~s}$. m.

Material estudiado (adicional al citado en Tortosa \& Bartoli, 2010): ARGENTINA: Neuquén: Dpto. Chos Malal, caj ón del arroyo del Cruce, faldeos $\mathrm{S}$ del Domuyo, O. Boelcke et al. 11286 (BAA); Dpto. Los Lagos, refugio cerro Colorado al cerro Tres Lagunas, J. Diem 1763 (BAA); Dpto. Minas, paso del Macho, O. Boelcke et al. 13912 (BAA); Dpto. Norquin, Copahue, F. Roig \& A. Ruiz Leal 22422 (LP). Río Negro: Dpto. Bariloche, cerro Rigi, $O$. Boelcke \& M. N. Correa 5461 (BAA); Dpto. 25 de Mayo, co. A necon Grande, E. Ferruglio 60 (LP). Chubut: Dpto. Fuatleufú, lago Futalaufquen, $A$. Soriano 4361 (BAA).

\subsection{SENECIO BIPONTINII SINÓNIMO DE $S$. CRITHMOIDES}

Cabrera (1949) diferenció a Senecio bipontinii Wedd. (sub. nom. S. julietii Phil., cf. Cabrera et al., 1999) de S. crithmoides Hook. et Arn., ambas de la serie Chilenses DC. ex M. G. López, A. F. Wulff \& Xifreda subserie Caespitosi (O. Hoffm.) Cabrera et S. E. Freire, por ser la primera más robusta (de 8-20 $\mathrm{cm}$ alt. vs. hasta $10 \mathrm{~cm}$. alt.), glauca y con las hojas enteras o con algún diente (vs. con 1 o 21 óbulos a cada lado), de margen revoluto. La variabilidad observada en los materiales estudiados nos hace considerar que ambos nombres corresponden a una única especie.
Senecio crithmoides Hook. et Arn., J. Bot. [Hook.] 3: 347. 1841. LECTOTIPO, aquí designado: Argentina. Mendoza: both sides of the cumbre from Las Cuevas to Piedra Puñal, III-1921, Gillies s. $n$. (K [K000497971], imagen digital!).

= Senecio sarcophyllus Phil., Linnaea 28: 740. 1856, nom. illeg. (Non Steud., 1841). TIPO : Chile. Volcán Osorno, II-1852, R. A. Philippi s. n. (holotipo, SGO, fotografía LP!). Syn. nov.

= Senecio schultzii Wedd., Chlor. Andina: 119. 1856, nom. illeg. (non Hochst., 1850). Senecio bipontinii Wedd., Chlor. Andina 1: 231. 1857, nuevo nombre para Senecio schultzii Wedd. Senecio carnifolius Cabrera, Notas Prelim. Mus. La Plata 3 (1): 114. 1934, nuevo nombre para Senecio schultzii Wedd. TIPO: Chile. In C ordillera ad 3000-3500', III-1852, R. A. Philippi 121 (isotipo, K, [K000497956], imagen digital!). Syn. nov.

= Senecio diazii Phil., Anales Univ. Chile 21: 399. 1862. TIPO: Argentina. Portillo, lado de Men doza, 1861/62, W. Diaz s. n. (holotipo, SGO; isotipo, LP [LP002373], SI [SI001038]).

= Senecio julietii Phil., Anales Univ. Chile 43: 496. 1873. TIPO: Chile. Volcán Calbuco, I- 1872, Juliet s. n. (holotipo, SGO, fotografía LP!).

= Senecio iberidifolius Phil., Anales Univ. Chile 88: 21. 1894. LECTOTIPO, aquí designado: Chile. Nuble: Termas de Chillán, II-1892, R. A. Philippis. n. (SGO, f otografía LP!, isolectotipo LP [LP002397]!.

= Senecio remyanus Phil., Anales Univ. Chile 88: 31. 1894. TIPO: Chile. Coquimbo: Quebrada del Pasto, 12-II-1883, F. Philippi (holotipo SGO; isotipo SI [SI001033]!).

Sufrútice rizomatoso, de 0, 08-0,2 m alt., glabro, formando céspedes densos. Tallos ascendentes, ramosos y densamente hojosos en la base, casi escapiformes en la parte $\mathrm{s}$ uperior. Hojas sésiles, crasas, con lámina elíptico-espatulada, de 10-30 X 2-7 mm, entera o con 1-2 lóbulos a cada lado cerca del ápice, obtusas. Capítulos discoides, solitarios en el extremos de las ramas. Involucro acampanado, caliculado, de 10-12 X 10-12 mm; bracteolas del calículo pocas, lineares, cortas. Filarias 12-20, linear-elípticas, glabras ápice agudo y penicelado. 


\section{R. D. Tortosa y A. Bartoli. - Novedades en el género Senecio}

Flores numerosas, amarillas. Aquenios glabros. Papus blanco.

Iconografia: Cabrera, 1949: 220, fig. 75 (sub. S. julietti); Cabrera, 1971: 224, fig. 225 (s ub. S. julietti), : 227, fig. 229 (sub. S. crithmoides).

Distribución y hábitat. Centro de Chile y Argentina, desde Mendoza y San Juan a Chubut, $1750-4500 \mathrm{~m}$.

Material adicional examinado: ARGENTINA. Mendoza: Dpto. Malargüe, Valle Hermoso, costa N de la Laguna del Valle, D. Medan 697 (BAA). Dpto. San Carlos, Laguna Diamante, El Paramillo, Boelcke 10052 y 10057 (BAA). Neuquén: Dpto. Catán Lil, Sierra de Chachil, Z. Rúgolo y E. Agrasar 424 (BAA). Dpto. Chos Malal, Riscos Bayos, O. Boelcke 11205 (BAA, LP). Dpto. Huiliches, Potrero del Volcán, A. Tarak s. n. (BAA). Dpto. Lacar, cerro Chapelco, San Martín de los Andes, R. León y $C$. Calderón s. n. (BAA 852); Cerro Malo, Z. Rúgolo y E. Agrasar 316 (BAA); Cerro Repollo, Z. Rúgolo y E. Agrasar 565 (BAA). Dpto. Los Lagos, refugio Cerro Colorado, O. Boelcke y M. N. Correa 6898 (BAA). Dpto. Minas, sierra de Cochicó, O. Boelcke 14074 (BAA). Río Negro: Dpto. Bariloche, cerro Catedral, H. A. Fabris 2235 (LP); Cerro Tronador, J. J. Valla et al s.n. (BAA 21135). Chubut: Dpto. Languiñeo, Tecka, Ea. Quichaura, A. Soriano 2296 (LP).

\section{AgRADECIMIENTOS}

Agradecemos a los curadores de K, LP, SGO y SI por la ac cesibilidad de los materiales, a la a rtista Norma Valgañon por las ilustraciones y a la Universidad de Buenos Aires y al Consejo Nacional de Investigaciones Científicas y Técnicas, Argentina, por el apoyo financiero.

\section{BIBLIOGRAFÍA}

CABRERA, A. L. 1949. El género Senecio en Chile. Lilloa 15: 27-501.

CABRERA, A. L. Compositae. 1971. En: M. N. Correa (ed.), Flora Patagónica parte 7: 5-451. INTA, Buenos Aires.

CABRERA, A. L., S. E. FREIRE \& L. ARIZA ESPINAR. 1999. Asteraceae, tribu VIII, Senecioneae. En: A. T. Hunziker (ed.), Flora Fanerogámica Argentina 62: 3-164. PROFLORA, CONICET.

HOSSEUS, C. C. 1915. La vegetación del lago Nahuel Huapi y sus montañas. Trab. Inst. Bot. Farmacol. 33: 1-102.

IUCN. 2001. IUCN Red List Categories and Criteria, Version 3.1. Prepared by the IUCN Species Survival Commission. IUCN, Gland, Switzerland, and Cambridge, UK.

PERÓN, J. D. 2000. Toponimia patagónica de etimología araucana. El Calafate Editores, Olivos, Provincia de Buenos Aires.

TORTOSA, R. D. \& A. BARTOLI. 2010. Consideraciones taxonómicas en especies de Senecio de Argentina. Bol. Soc. Argent. 45: 373-381.

Recibido el 10 de diciembre de 2013, aceptado el 9 de mayo de 2014. 